\title{
Brevundimonas vesicularis Peritonitis in a Chronic Peritoneal Dialysis Patient
}

\author{
Vijayakumar Paramasivam ${ }^{a} \quad$ Armando Paez $^{b} \quad$ Ashish Verma $^{c}$ \\ Daniel Landryc Gregory L. Braden ${ }^{c}$ \\ aDivision of Hospital Medicine, Baystate Medical Center, University of Massachusetts \\ Medical School at Baystate, Springfield, MA, USA; bivision of Infectious Disease, Baystate \\ Medical Center, University of Massachusetts Medical School at Baystate, Springfield, \\ MA, USA; 'Division of Nephrology, Baystate Medical Center, University of Massachusetts \\ Medical School at Baystate, Springfield, MA, USA
}

\section{Keywords}

Peritonitis · Peritoneal dialysis · Brevundimonas

\section{Abstract}

Gram-negative peritonitis in chronic peritoneal dialysis patients is difficult to treat and may result in catheter loss. Brevundimonas vesicularis is a Gram-negative rod bacterium which rarely causes infections in humans. A 41-year-old male receiving continuous cycling peritoneal dialysis for 5 months developed culture-negative peritonitis. He failed initial empiric treatment with intraperitoneal vancomycin and levofloxacin and thereafter intravenous gentamicin. $B$. vesicularis resistant to levofloxacin was isolated from the peritoneal fluid 21 days after his initial symptoms. Despite treatment with intravenous ceftriaxone and oral amoxicillin-clavulanate, the infection persisted, which required removal of the peritoneal catheter in order to cure this infection. We describe the features of $B$. vesicularis infection in our patient and the rarely reported additional cases.

\section{Introduction}

Brevundimonas (formerly Pseudomonas) vesicularis is an aerobic nonsporulating, slender, motile, nonlactose fermenting Gram-negative rod that has been infrequently isolated from the external environment and human clinical specimens. This has been previously reported 
as a cause of nosocomial infections and is one of the emerging global opportunistic pathogens. We report the 5th case of peritonitis caused by Brevundimonas vesicularis in a patient on continuous cycling peritoneal dialysis (CCPD). We will review the epidemiology, clinical presentations, and diagnostic and therapeutic challenges of human infections caused by this organism.

\section{Case Report}

A 41-year-old white male developed diffuse abdominal pain and cloudy peritoneal dialysate. He had end-stage kidney disease from hypertensive nephrosclerosis and had been on CCPD since November 2003. Between 5 and 6 p.m. everyday, he would perform a pause exchange of $2 \mathrm{~L}$ in order to empty his long dwelling peritoneal fluid during the day. His medications included irbesartan, nephrocaps, pyridoxine, atorvastatin, polyethylene glycol, and methadone as needed for pain. He was not an IV drug abuser. His daily exit site care included a soap and water wash followed by mupirocin ointment. He was renovating a humid, moldy basement for a month before developing abdominal pain. He denied performing a dialysis exchange in the basement.

Five months after initiating CCPD, in April 2004, he developed clinical symptoms of peritonitis with a peritoneal dialysate WBC count of $333 \mathrm{k} / \mathrm{mm}^{3}$, with $55 \%$ neutrophils and $30 \%$ monocytes. He was given vancomycin $2 \mathrm{~g}$ and levofloxacin $500 \mathrm{mg}$ intraperitoneally (IP) with a 6-h dwell. Oral levofloxacin $250 \mathrm{mg}$ daily and clotrimazole troches 3 times a day were also prescribed. He returned 3 days later for another dose of IP vancomycin. The initial peritoneal fluid Gram stain and culture at a local hospital laboratory were negative. Six days later, he still had cloudy peritoneal fluid, and the cultures grew an unidentified Gram-negative rod from a national microbiology laboratory, Spectra. IP vancomycin $2 \mathrm{~g}$ every 5 days and oral levofloxacin $250 \mathrm{mg}$ daily were continued. Thirteen days after his initial presentation, he developed increasing abdominal pain. Physical examination revealed diffuse abdominal pain with mild rebound tenderness. Repeat WBC peritoneal fluid count was $225 \mathrm{k} / \mathrm{mm}^{3}$, and peritoneal fluid Gram stain showed Gram-negative rods. He was started on IP tobramycin in addition to IP levofloxacin $250 \mathrm{mg}$ daily. Tobramycin was added to the peritoneal dialysis fluid to a final concentration of $5 \mathrm{mcg} / \mathrm{mL}$ during CCPD. Twentyone days after initial presentation, $B$. vesicularis was finally identified in 2 peritoneal fluid cultures from days 6 to 13 of his illness. Both B. vesicularis isolates were susceptible to amikacin, cefotaxime, ceftriaxone, colistimethate, gentamicin, imipenem, tobramycin, and ticarcillin/clavulanate. Both isolates were resistant to levofloxacin and ciprofloxacin. He was subsequently admitted to the hospital and treated with ceftriaxone $2 \mathrm{~g}$ IV daily for 3 days. The peritoneal catheter was not initially removed. At discharge, his antibiotic was changed to oral amoxicillin/clavulanate $500 \mathrm{mg}$ twice daily. Nine days later, he developed abdominal pain and cloudy peritoneal fluid, and he was readmitted to the hospital with the peritoneal fluid WBC count of $666 \mathrm{k} / \mathrm{mm}^{3}$. Ceftriaxone $2 \mathrm{~g}$ IV daily was reinitiated, but the peritoneal fluid WBC count increased to $1,010 \mathrm{k} / \mathrm{mm}^{3}$ on day 3 , and the peritoneal catheter was surgically removed due to continued abdominal symptoms and the rising peritoneal dialysate WBC. The peritoneum was erythematous and diffusely inflamed. Intraoperative bacterial and fungal cultures were negative. The catheter tip culture was also negative. The patient was temporarily switched to hemodialysis and received ceftriaxone $2 \mathrm{~g}$ IV daily for a total of 12 days with complete resolution of symptoms. Four weeks later, a peritoneal dialysis catheter was successfully replaced, and the patient resumed CCPD for an additional 6 months. Subsequently, after an episode of Staphylococcus aureus peritonitis in December 2004, he was permanently changed to chronic hemodialysis.

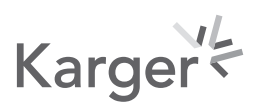




\section{Discussion}

To our knowledge, this report is the 5th case of acute peritonitis from B. vesicularis and the 1st case in dialysis patients reported to have resistance to quinolones (Table 1). The clinical course of our patient identifies several unique characteristics of this infection in chronic peritoneal dialysis patients. Four out of the 5 reported patients had to have peritoneal dialysis catheter removal in order to eradicate the infection [1-4]. Also, 4 out of the 5 reported patients had eventual recovery, but 1 patient developed encapsulating sclerosing peritonitis (Table 1). Our patient returned to successful peritoneal dialysis for 6 months. Three out of the 5 reported cases had to be switched to hemodialysis after peritoneal dialysis catheter removal for $B$. vesicularis peritonitis [1-3].

$B$. vesicularis represents a separate line in the $\alpha$-subclass of Proteobacteria, rarely encountered in human infection. It was first described in 1953 by Busing et al. [5], who cultured it from the seminal vesicle of a medicinal leech and initially gave it the name Corynebacterium vesiculare. In 1994, Segers et al. [6] reclassified 2 species originally described as Pseudomonas diminuta and Pseudomonas vesicularis into a new genus Brevundimonas, based on DNA-rRNA hybridization studies, 16S rRNA cataloging, and 16S rRNA sequencing. In the microbiology laboratory, the bacteriologic features characteristic of this organism include production of either a tan-brown water-soluble pigment or a yellow or orange growth pigment and the requirement of special growth factors including pantothenate, biotin, and cyanocobalamin. It is oxidase positive and can give variable results. Only about $25 \%$ of B. vesicularis grow on MacConkey agar. Many strains of $B$. vesicularis have orange color due to an intracellular pigment. This may be correctly identified by automated microbiology systems depending on the type used by the local laboratory. If this organism is suspected based on biochemical properties and the local laboratory has limited ability to identify it, the isolate should be sent to a reference laboratory for further workup and identification. The antibiotic therapy for our patient was hindered by the inability of 2 independent microbiology laboratories to successfully identify $B$. vesicularis as a true human pathogen.

The initial empiric antibiotic therapy in our case failed to adequately treat Brevundimonas peritonitis. In 2004, the International Society of Peritoneal Dialysis (ISPD) guidelines for empiric therapy for peritonitis suggested IP cefazolin for Gram-positive coverage, and ceftazidime for Gram-negative coverage. Our facility at that time utilized vancomycin rather than cefazolin because our local antibiogram reported a high incidence of methicillin-resistant S. aureus. Levofloxacin was utilized because it was a recommended empiric treatment for Gram-negative organisms as an alternative to 3rd-generation cephalosporin, and it could be given orally in follow-up [4, 7-9]. Since B. vesicularis in our patient was sensitive to ceftriaxone and cefotaxime, it is possible that if we had used IP ceftazidime from the time of treatment initiation, the patient may have had a better outcome. Indeed in 2006, we changed our protocol to IP ceftazidime for Gram-negative coverage, and IP vancomycin remained to cover Gram-positive organisms. However, empiric aminoglycosides have not been included in our initial treatment protocol due to their potential nephrotoxic effects on residual renal function $[10,11]$. In contrast, recent data suggest that short-term use of aminoglycosides in chronic dialysis patients appears to be safe and does not diminish residual renal function [12]. This is reflected in the 2005 and 2016 guidelines for empiric therapy for peritonitis by the International Society of Peritoneal Dialysis (ISPD) $[13,14]$. When our usual antibiotic regimen failed after 6 days, broader antibiotic coverage with aminoglycosides should have been initiated, but this did not occur until 13 days after the onset of illness. B. vesicularis cultured in our patient was sensitive to gentamicin and tobramycin but not to levofloxacin and ciprofloxacin. A delay in the aminoglycoside administration may have contributed to the refractory peritonitis in our patient. Once our patient received high doses of intravenous

\section{Karger'}


Case Reports in Nephrology and Dialysis
Case Rep Nephrol Dial 2021;11:314-320

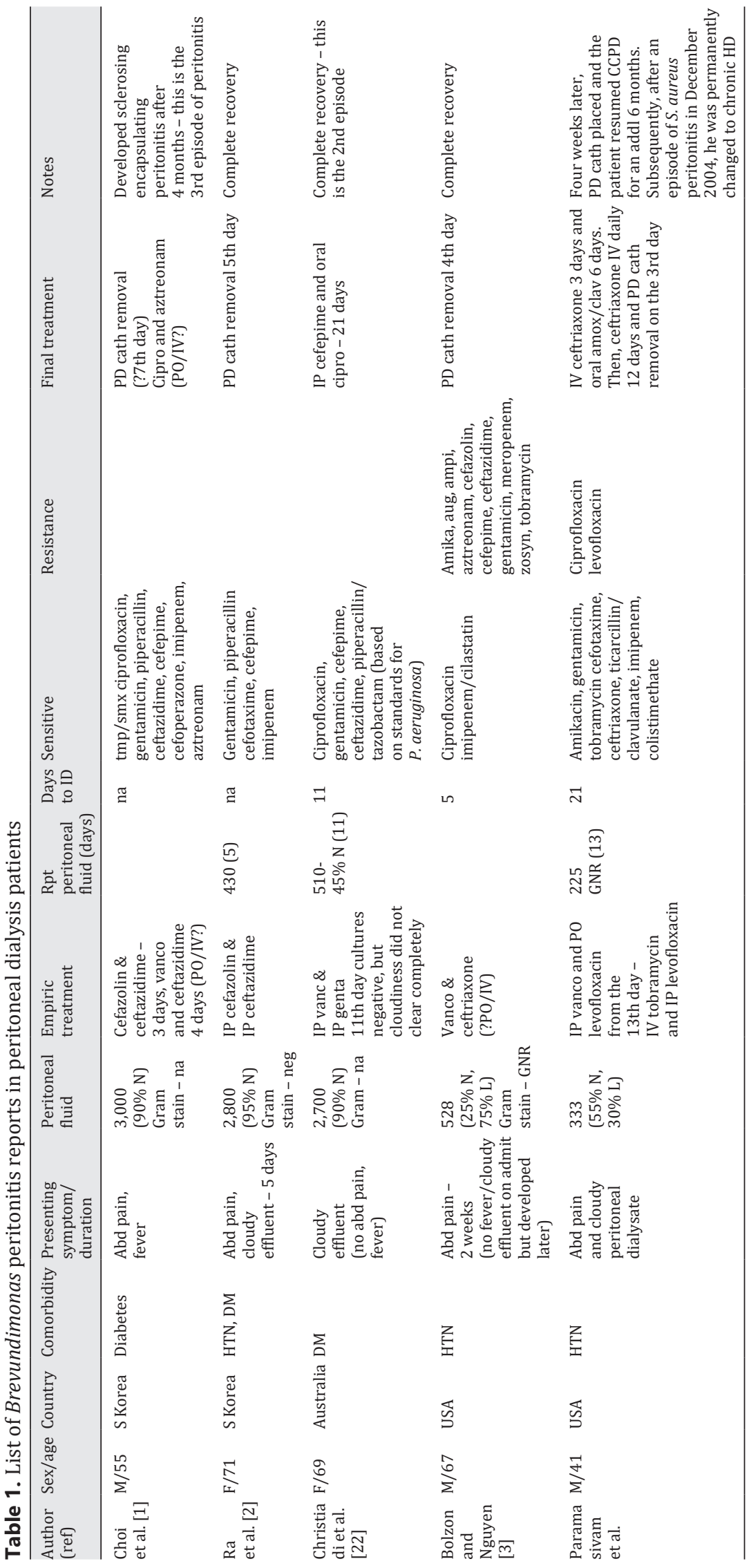


ceftriaxone, his peritoneal fluid WBC became normal, and his abdominal symptoms abated. However, 9 days of amoxicillin-clavulanic acid oral therapy suppressed this infection, but he unfortunately suffered a severe relapse. Amoxicillin-clavulanic acid was selected on the basis of previous case reports published prior to our case $[15,16]$.

Refractory peritonitis in chronic peritoneal dialysis patients often necessitates peritoneal dialysis catheter removal due to infection with bacteria that produce biofilm, which coats the catheter with slime in which bacteria are embedded. This antibiotic-resistant environment of exopolysaccharides prevents eradication of the infection. Several studies in paper mills have confirmed that $B$. vesicularis produces exopolysaccharides which form biofilm [1719]. We believe that biofilm production by B. vesicularis occurred in 4 of the 5 reported patients and contributed to the failure to eradicate these infections until the peritoneal dialysis catheter was removed.

It is possible that our patient contracted $B$. vesicularis from working in a basement environment. The basement was cool and humid with large amounts of mold that had to be removed before new walls could be constructed. We did not test if $B$. vesicularis was present in the basement. However, this organism has been isolated from various water-related equipment such as tap water aerators, hospital sinks, water taps and water tanks, dental unit reservoirs, reused dialyzers, bottled mineral water, corneal specimens, and contact lenses. It is possible that improper sterile technique or cleansing by the patient in this environment may have allowed accidental exposure to this organism.

A review of the literature reveals that this organism has rarely been implicated in human infections. $B$. vesicularis has been recovered from human specimens including cerebrospinal fluid, cervical secretions, eye, urine, and wound cultures [20]. B. vesicularis is associated with a wide range of human infections including cellulitis, pneumonia, septicemia, tonsillitis, endocarditis, septic arthritis, meningitis, skin infections, keratitis, and peritonitis.

Antimicrobial resistance of this organism to antibiotics often used to treat Gram-negative infections is common [21]. B. vesicularis isolates in our case were found to be resistant to levofloxacin and ciprofloxacin, but susceptible to 3rd-generation cephalosporins and aminoglycosides. However, resistance to 3rd-generation cephalosporins and aminoglycosides has been reported in other cases [21]. It is difficult to recommend the best empiric therapy for this organism because of variable antimicrobial resistance. There is only limited clinical experience in treating this infection based on drug resistance results.

\section{Conclusion}

In summary, B. vesicularis peritonitis in chronic peritoneal dialysis patients appears to be refractory to antibiotic therapy alone. Prompt removal of the peritoneal dialysis catheter in addition to appropriate antibiotic therapy likely is necessary to cure this infection. Clinicians should be aware of this rare infection in chronic peritoneal dialysis patients. Laboratories supporting the care of peritoneal dialysis patients should be able to correctly identify $B$. vesicularis.

\section{Statement of Ethics}

This study was performed adhering to all guidelines adopted by the World Medical Association Declaration of Helsinki-Ethical Principles for Medical Research involving Human Subjects. The patient has given us written consent for this publication. All data utilized in this study were deidentified.

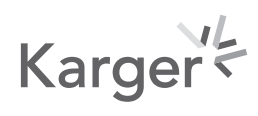




\section{Conflict of Interest Statement}

The authors have no conflicts of interest or disclosures of any kind.

\section{Funding Sources}

This is a simple case report from medical records, no funding was required.

\section{Author Contributions}

All authors made substantial contributions to the conception or design of the work or the acquisition, analysis, or interpretation of data for the work. All authors contributed to drafting the work or revising it critically for important intellectual content. All authors gave final approval of the version to be published. All authors are in agreement to be accountable for all aspects of the work in ensuring that questions related to the accuracy or integrity of any part of the work are appropriately investigated and resolved.

\section{References}

1 Choi W, Lee C, Kim A, Choi JW, Seo S, Lee J, et al. CAPD peritonitis due to Brevundimonas vesicularis. Perit Dial Int. 2006;26(4):510-2.

2 Ra H, Ahn JH, Ho J, Song JS, Yoon HJ, Sun IO, et al. S-337: a case of peritoneal dialysis-associated peritonitis caused by Brevundimonas vesicularis. Kor J Inter Med. 2015;337:206.

3 Bolzon C, Nguyen BH. A rare case of peritonitis due to Brevundimonas vesicularis. J Community Hosp Intern Med Perspect. 2018;8(3):161-2.

4 Keane WF, Bailie GR, Boeschoten E, Gokal R, Golper TA, Holmes CJ, et al. Adult peritoneal dialysis-related peritonitis treatment recommendations: 2000 update. Perit Dial Int. 2000;20(4):396-411.

5 Busing KH, Doll W, Freytag K. Bacterial flora of the medicinal leech. Arch Mikrobiol. 1953;19(1):52-9.

6 Segers P, Vancanneyt M, Pot B, Torck U, Hoste B, Dewettinck D, et al. Classification of Pseudomonas diminuta Leifson and Hugh 1954 and Pseudomonas vesicularis Busing, Doll, and Freytag 1953 in Brevundimonas gen. Nov. As Brevundimonas diminuta comb. Nov. And Brevundimonas vesicularis comb. Nov., Respectively. Int J Syst Bacteriol. 1994;44(3):499-510.

7 Zelenitsky S, Barns L, Findlay I, Alfa M, Ariano R, Fine A, et al. Analysis of microbiological trends in peritoneal dialysis-related peritonitis from 1991 to 1998. Am J Kidney Dis. 2000;36(5):1009-13.

8 Cheng IK, Fang GX, Chau PY, Chan TM, Tong KL, Wong AK, et al. A randomized prospective comparison of oral levofloxacin plus intraperitoneal (IP) vancomycin and IP netromycin plus IP vancomycin as primary treatment of peritonitis complicating CAPD. Perit Dial Int. 1998;18(4):371-5.

9 Lye WC, Lee EJ, van der Straaten J. Intraperitoneal vancomycin/oral pefloxacin versus intraperitoneal vancomycin/gentamicin in the treatment of continuous ambulatory peritoneal dialysis peritonitis. Perit Dial Int. 1993;13 Suppl 2:S348-50.

10 Singhal MK, Bhaskaran S, Vidgen E, Bargman JM, Vas SI, Oreopoulos DG. Rate of decline of residual renal function in patients on continuous peritoneal dialysis and factors affecting it. Perit Dial Int. 2000;20(4):429-38.

11 Shemin D, Maaz D, St Pierre D, Kahn SI, Chazan JA. Effect of aminoglycoside use on residual renal function in peritoneal dialysis patients. Am J Kidney Dis. 1999;34(1):14-20.

12 Lui SL, Cheng SW, Ng F, Ng SY, Wan KM, Yip T, et al. Cefazolin plus netilmicin versus cefazolin plus ceftazidime for treating CAPD peritonitis: effect on residual renal function. Kidney Int. 2005;68(5):2375-80.

13 Piraino B, Bailie GR, Bernardini J, Boeschoten E, Gupta A, Holmes C, et al. ISPD Ad Hoc Advisory Committee. Peritoneal dialysis-related infections recommendations: 2005 update. Perit Dial Int. 2005;25(2):107-31.

14 Li PK, Szeto CC, Piraino B, de Arteaga J, Fan S, Figueiredo AE, et al. ISPD peritonitis recommendations: 2016 update on prevention and treatment. Perit Dial Int. 2016;36(5):481-508.

15 Chi CY, Fung CP, Wong WW, Liu CY. Brevundimonas bacteremia: two case reports and literature review. Scand J Infect Dis. 2004;36(1):59-61.

16 Panasiti V, Devirgiliis V, Mancini M, Curzio M, Rossi M, Fioriti D, et al. A cutaneous infection caused by Brevundimonas vesicularis: a case report. Int J Immunopathol Pharmacol. 2008;21(2):457-61.

17 Ratto M, Suihko ML, Siika-aho M. Polysaccharide-producing bacteria isolated from paper machine slime deposits. J Ind Micobiol Biotechnol. 2005;32(3):109-14. 
18 Verhoef R, de Waard P, Schols HA, Rättö M, Siika-aho M, Voragen AG. Structural elucidation of the EPS of slime producing Brevundimonas vesicularis sp. isolated from a paper machine. Carbohydr Res. 2002;337(20): 1821-31.

19 Verhoef R, Schols HA, Blanco A, Siika-aho M, Rättö M, Buchert J, et al. Sugar composition and FT-IR analysis of exopolysaccharides produced by microbial isolates from paper mill slime deposits. Biotechnol Bioeng. 2005; 91(1):91-105.

20 Clark WA, Hollis DG, Weaver RE, Riley P. Identification of unusual pathogenic gram-negative aerobic and facultatively anaerobic bacteria. Atlanta, GA: Centers for disease control, US Department of Health and human services; 1985.

21 Shang ST, Chiu SK, Chan MC, Wang NC, Yang YS, Lin JC, et al. Invasive Brevundimonas vesicularis bacteremia: two case reports and review of literature. J Microbiol Immunol Infect. 2012;45(6):468-72.

22 Christiadi D, Singer RF, Roberts DM. Successful treatment of PD peritonitis due to Brevundimonas vesicularis. Perit Dial Int. 2018 Sep-Oct;38(5):379-81. 